\title{
Bandwidth Allocation with Minimum Rate Constraints in Cluster-based Femtocell Networks
}

\author{
Kai Ma ${ }^{1} \quad$ Liang $\mathrm{Li}^{1} \quad$ Jie Yang $^{1} \quad$ Zhi-Xin Liu ${ }^{1} \quad$ Xin-Bin $\mathrm{Li}^{1} \quad$ Xin-Ping Guan ${ }^{1,2}$ \\ ${ }^{1}$ School of Electrical Engineering, Key Laboratory of Industrial Computer Control Engineering of Hebei Province, Yanshan University, \\ Qinhuangdao 066004, China \\ ${ }^{2}$ Department of Automation, Shanghai Jiao Tong University, Key Laboratory of System Control and Information Processing, Ministry of \\ Education, Shanghai 200240, China
}

\begin{abstract}
Inter-femtocell interference becomes serious when femtocells are densely deployed. To mitigate the inter-femtocell interference, this paper proposes a cluster-based bandwidth allocation algorithm. We create femtocell clusters by constructing a weighted interference graph and allocate bandwidth to each cluster based on a Nash bargaining solution (NBS). Simulation results show that the cluster-based bandwidth allocation algorithm can reduce the inter-femtocell interference and meet the minimum rate constraint of each cluster.
\end{abstract}

Keywords: Femtocell, cluster, inter-femtocell interference, bandwidth allocation, Nash bargaining solution (NBS).

\section{Introduction}

With the development of wireless communications, it becomes more and more important to make full use of scarce spectrum resource. Future wireless mobile communication system is to provide better service for users, guarantee quality of service, and support more reliable transmission. At the same time, it should also provide higher data rate and spectrum utilization. As reported in [1], 70\% data services and more than $50 \%$ voice services take place in the indoor environment. Thus, it is necessary to enhance the indoor coverage and connect the data services with voice services more efficiently.

Femtocell, also named home base station, is a shortrange, low-cost, and low-power wireless data access point installed by the consumers inside home or buildings for better indoor voice and data service. Femtocell is connected to the mobile operator's network by a physical broadband connection such as digital subscriber line ${ }^{[1]}$. It can expand coverage and increase spectral efficiency. Interference management is necessary for the application of femtocell. The interference in the femtocell network includes the interfemtocell interference among femtocells and the cross-tier interference between macrocell and femtocells.

In recent years, power control is used for mitigating

\footnotetext{
Regular paper
Manuscript received July 8, 2013; accepted February 14, 2014

This work was supported by National Basic Research Program of China (No. 2010CB731800), National Natural Science Foundation of China (Nos. 61203104, 61221003, 61174127, 61104033 and 61172095), Natural Science Foundation of Hebei Province (Nos. F2012203126 and F2012203109), Research Foundation for the Doctoral Program of Higher Education (Nos. 20121333120012, 20110073130005, and 20110073120025), and Program for Doctor Foundation of Yanshan University (No. B632).

Recommended by Associate Editor Shuang-Hua Yang

(C) Institute of Automation, Chinese Academy of Science and Springer-Verlag Berlin Heidelberg 2015
}

the cross-tier interference. In [2], a utility-based adaptive algorithm was proposed to reduce the cross-tier interference by regulating the signal-to-interference-plus-noise ratio (SINR). In [3], the authors proposed a distributed power control method based on Stackelberg game to mitigate the interference. In [4], the authors developed an opportunistic power control algorithm to mitigate the aggregate interference from active femtocells in each cluster. However, these works only deal with the cross-tier interference. In fact, the inter-femtocell interference is serious when the femtocell is densely deployed. To resolve this problem, some inter-femtocell interference mitigation schemes are proposed. In [5], the authors jointly considered the power and sub-channel allocation to reduce the inter-femtocell interference. A distributed dynamic interfemtocell interference mitigation scheme was studied in [6] to guarantee high SINR for each user. These works require information exchanges and increase the network overhead. A novel clustering scheme named power and coverage-aware clustering was proposed in [7]. In [8], the authors used a cluster head to determine the resource allocation within each cluster and resolved the collisions among clusters. The energy consumption of femtocell cluster head is high, and only local optimization is achieved by this method. In [9], the femtocells are divided into clusters, and the spectrum resource is allocated to each cluster. However, some femtocells cannot meet the minimum rate constraints because of the limited resource. Thus, it is necessary to allocate the bandwidth to meet the minimum rate constraint of each cluster.

In this paper, we propose a cluster-based bandwidth allocation algorithm (CBBAA) based on Nash bargaining solution (NBS) to mitigate the inter-interference in the femtocell network. The NBS was used for allocating time slots 
to the mobile users in cooperative relay networks [10]. In this study, we assume that the femtocells are densely deployed and divided into clusters according to a weighted interference graph. Then, the bandwidth is allocated to each cluster by NBS.

The rest of the paper is organized as follows. Section 2 establishes the network model. Section 3 constructs a weighted interference graph and develops a cluster-based bandwidth allocation algorithm using NBS. Section 4 gives the numerical results, and Section 5 concludes the paper.

\section{Network model}

We consider a two-tier cellular network, where the first tier is a macrocell and the second tier is composed of femtocells. The femtocells are randomly deployed in the coverage of the macrocell. In this study, we focus on the downlink transmission in the femtocell network based on orthogonal frequency-division multiple access (OFDMA), as shown in Fig. 1.

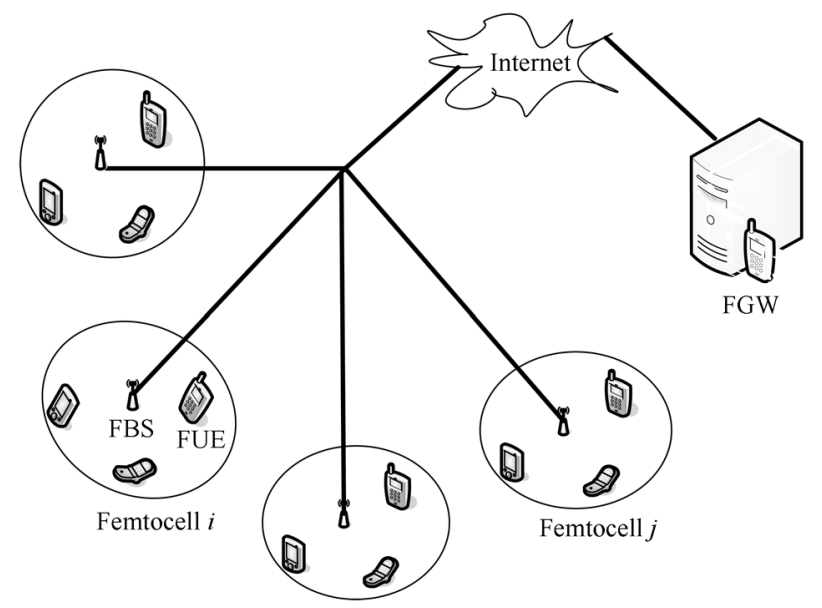

Fig. 1 Femtocell networks

The interference from the macrocell base station (MBS) is regarded as an additive white Gaussian noise. All femtocell base stations (FBS) are connected to a femtocell gateway (FGW), which manages the operation and maintenance (OAM) information such as the femtocell location and identification through a backhaul link. FGW also manages the spectrum resource in the femtocell network based on the OAM information. Let $\mathbb{N}=\{0,1, \cdots, N\}$ denote the set of femtoells. Without loss of generality, we assume only one femtocell user (FUE) is connected to the FBS in a femtocell. The transmission power of FBS $i(i \in \mathbb{N})$ is assumed to be fixed at $p_{i}$. The SINR of FUE $i$ can be denoted as

$$
r_{i}=\frac{p_{i} H_{i i}}{\sum_{j \neq i} p_{j} H_{i j}+\sigma^{2}}
$$

where $H_{i i}$ denotes the channel gain from FBS $i$ to FUE $i$, $H_{i j}$ denotes the channel gain from $\operatorname{FBS} j(j \in \mathbb{N}, j \neq i)$ to FUE $i$, and $\sigma^{2}$ is the single-sided spectral density of the independent white Gaussian noise.

\section{Bandwidth allocation algorithm}

In this section, we develop a bandwidth allocation algorithm for the OFDMA-based femtocell network. The cluster-based bandwidth allocation algorithm (CBBAA) can reduce the inter-femtocell interference and improve the spectrum efficiency. First, a weighted interference graph is constructed based on the topology of the femtocell network. Then, the femtocells are divided into several clusters. Finally, a bandwidth allocation algorithm is developed to meet the minimum rate constraint of each cluster.

\subsection{Weighed interference graph and clus- tering}

Based on the topology of the given femtocell network, we can construct a weighted interference graph $G=(V, E, W)$, where $V$ is the node set, $E$ is the bi-directional edge set, and $W$ is a weight matrix. We define $V=\left\{v_{1}, v_{2}, \cdots, v_{n}\right\}$, $E=\left\{e_{i j}, i, j \in\{0,1, \cdots, N\}, i \neq j\right\}$, and $W=\left\{w_{i j}, i, j \in\right.$ $\{0,1, \cdots, N\}, i \neq j\}$, where $v_{i}$ denotes the node corresponding to femtocell $i, e_{i j}$ is the edge between node $v_{i}$ and node $v_{j}$, and $w_{i j}$ is the weight of the edge $e_{i j}$. Then, the interference relationships among the femtocells are simplified to the connections among the nodes. Specifically, FUE $i$ is interfered by FBS $j$ when node $v_{i}$ and node $v_{j}$ are connected by a directed edge $e_{i j}$, and $w_{i j}$ denotes the interference strength.

The weight is determined by the FUE measurement report based on the reciprocal of SINR. We consider two femtocells $i$ and $j$ in a femtocell network, where FUE $i$ is interfered by $\mathrm{FBS} j$. Assuming that the transmission power of FBS $i$ and FBS $j$ are $p_{i}$ and $p_{j}$, respectively. Then, the weight $w_{i j}$ between femtocell $i$ and femtocell $j$ is denoted as

$$
w_{i j}= \begin{cases}\frac{p_{j} H_{i j}+\sigma^{2}}{p_{i} H_{i i}}, & \frac{p_{j} H_{i j}+\sigma^{2}}{p_{i} H_{i i}} \geq \delta \\ 0, & \frac{p_{j} H_{i j}+\sigma^{2}}{p_{i} H_{i i}}<\delta\end{cases}
$$

where $\delta$ is the interference threshold. The weight is zero when the interference among femtocells is lower than the threshold. Then, the femtocells are divided into clusters based on the weights. We assume the number of clusters is $M$ and denote the set of clusters as $\mathbb{M}=\{1,2, \cdots, M\}$. The interference strength of femtocell $i$ is calculated as $w_{i}=$ $\sum_{j \neq i} w_{i j}$. The set of femtocells in cluster $m(m \in \mathbb{M})$ is denoted as $\mathbb{N}_{m}=\left\{1,2, \cdots, N_{m}\right\}$, where $N_{m}$ is the number of femtocells in cluster $m$. The interference strength between femtocell $i$ and cluster $m$ is calculated as $w_{i}^{m}=\sum_{j \in \mathbb{N}_{m}} w_{i j}$. We first calculate the interference strength $w_{i}$ for all femtocells and sort $w_{i}$ of a sequence in descending order. Then, the first $M$ femtocells in the sequence are assigned into the $M$ clusters, respectively. The rest of femtocells are assigned to the cluster with the minimal $w_{i}^{m}$ one after another. When more than one cluster has the same minimal $w_{i}^{m}$, the femtocell is assigned to one of them randomly. The clustering algorithm is given in Fig. 2. 


\subsection{Bandwidth allocation based on NBS}

In this section, we will allocate bandwidth to each cluster based on NBS. Next, we first give the definitions of NBS and Pareto optimality ${ }^{[11,12]}$.

Definition 1. Let $\mathbb{M}=\{1,2, \cdots, M\}$ denote the set of players and $S$ denote the set of feasible payoff allocations, which should be a closed and convex set on $R^{M}$. $E^{\mathrm{min}}=\left(E_{1}^{\mathrm{min}}, \cdots, E_{m}^{\mathrm{min}}, \cdots, E_{M}^{\min }\right)$, where $E_{m}^{\min }$ denotes minimum payoff of player $m$. Then, $\left(S, E^{\text {min }}\right)$ is a $M$-player bargaining problem.

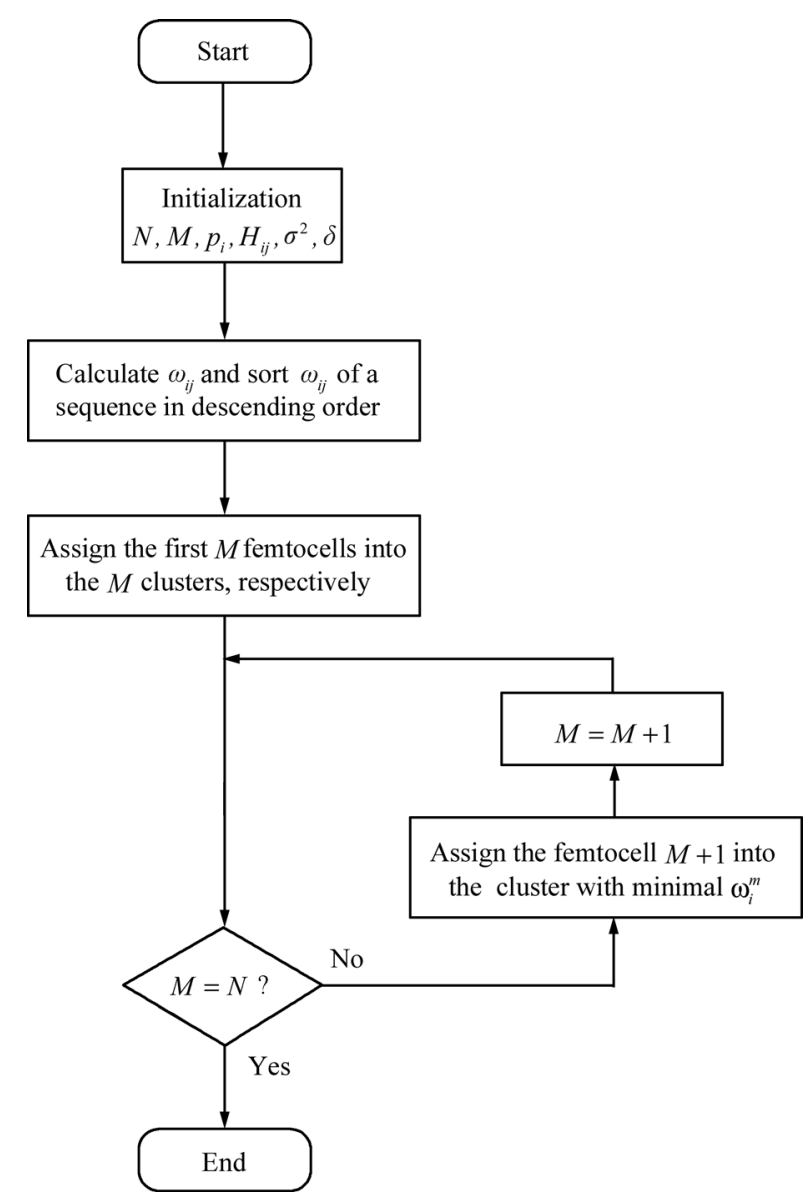

Fig. 2 The clustering algorithm

Within the feasible set $S$, we define the notion of Pareto optimality.

Definition 2. The payoff allocation $E=\left(E_{1}, \cdots, E_{M}\right)$ is Pareto optimal if and only if there is no other allocation $E_{m}^{\prime}$ such that $E_{m}^{\prime}>E_{m}, \forall m$, i.e., there exists no other allocation that leads to superior performance for some player without inferior performance for some other player.

The bargaining problem $\left(S, E^{\mathrm{min}}\right)$ has many Pareto optimal solutions denoted by $f\left(S, E^{\mathrm{min}}\right)$. A critical criterion is the fairness of resource allocations. In this study, we employ NBS with proportional fairness. Assuming that the minimum payoff is $E_{m}^{\min }$. Then, the NBS can be defined as the solution of the following optimization problem.

$$
E_{m}^{*}=\arg \max _{E_{m} \in S} \prod_{m=1}^{M}\left(E_{m}-E_{m}^{\mathrm{min}}\right) .
$$

We assume the allocated fraction of bandwidth for cluster $m$ is $\alpha_{m}, 0 \leq \alpha_{m} \leq 1$. The sum of the fractions should satisfy $\sum_{m=1}^{M} \alpha_{m} \leq 1$. Thus, the set of feasible payoff allocations can be denoted as

$$
\begin{gathered}
Q=\left\{\Gamma=\left(\Gamma_{1}, \cdots, \Gamma_{m}, \cdots, \Gamma_{M}\right) \mid 0 \leq \alpha_{m} \leq 1\right. \\
\left.\sum_{m=1}^{M} \alpha_{m} \leq 1, m=1,2, \cdots, M\right\}
\end{gathered}
$$

where $\Gamma_{m}$ is the transmission rate of cluster $m$.

$$
\Gamma_{m}=\alpha_{m} W \sum_{i \in \mathbb{N}_{m}} \ln \left(1+r_{i}\right)
$$

where $W=\frac{\alpha W_{0}}{\ln 2}, \alpha$ is the gap between the achievable rate and the actual transmission rate, and $W_{0}$ is the total transmission bandwidth for the femtocell network. Next, we will prove $Q$ is a convex subset of $R^{M}$. According to the rate function (5) of cluster $m, Q$ is closed subset on $R^{M}$. Assuming that $\Gamma^{a}=\left(\Gamma_{1}^{a}, \cdots, \Gamma_{m}^{a}, \cdots, \Gamma_{M}^{a}\right) \in Q$, $\Gamma^{b}=\left(\Gamma_{1}^{b}, \cdots, \Gamma_{m}^{b}, \cdots, \Gamma_{M}^{b}\right) \in Q$, and $0 \leq \theta \leq 1$, we have

$$
\theta \Gamma_{m}^{a}+(1-\theta) \Gamma_{m}^{b}=\left[\theta \alpha_{m}^{a}+(1-\theta) \alpha_{m}^{b}\right] W \sum_{i \in \mathbb{N}_{m}} \ln \left(1+r_{i}\right) .
$$

According to $\Gamma_{m}^{a} \in Q$ and $\Gamma_{m}^{b} \in Q$, we have $0 \leq \alpha_{m}^{a} \leq 1$, $0 \leq \alpha_{m}^{b} \leq 1, \sum_{m=1}^{M} \alpha_{m}^{a} \leq 1$, and $\sum_{m=1}^{M} \alpha_{m}^{b} \leq 1$. Then, we obtain

$$
0 \leq \theta \alpha_{m}^{a}+(1-\theta) \alpha_{m}^{b} \leq 1
$$

and

$$
\sum_{m=1}^{M}\left(\theta \alpha_{m}^{a}+(1-\theta) \alpha_{m}^{b}\right)=\theta \sum_{m=1}^{M} \alpha_{m}^{a}+(1-\theta) \sum_{m=1}^{M} \alpha_{m}^{b} \leq 1 .
$$

Then, we can conclude that $Q$ is closed and convex on $R^{M}$. The net rate function of cluster $m$ can be denoted as

$$
U_{m}=\Gamma_{m}-\Gamma_{m}^{\min }
$$

where $\Gamma_{m}^{\min }$ is the minimum rate of cluster $m$. Then, the NBS-based bandwidth allocation problem can be denoted as

$$
\begin{array}{ll}
\max & \prod_{m=1}^{M} U_{m} \\
\text { s.t. } & \sum_{m=1}^{M} \alpha_{m} \leq 1 .
\end{array}
$$

The optimization problem (7) is equivalent to solving the following optimization problem.

$$
\begin{array}{ll}
\max & \sum_{m=1}^{M} \ln U_{m} \\
\text { s.t. } & \sum_{m=1}^{M} \alpha_{m} \leq 1 .
\end{array}
$$


The first and second derivative of $\ln U_{m}$ with respect to $\alpha_{m}$ can be denoted as

$$
\frac{\mathrm{d} \ln U_{m}}{\mathrm{~d} \alpha_{m}}=\frac{W \sum_{i \in \mathbb{N}_{m}} \ln \left(1+r_{i}\right)}{\Gamma_{m}-\Gamma_{m}^{\min }}>0
$$

and

$$
\frac{\mathrm{d}^{2} \ln U_{m}}{\mathrm{~d} \alpha_{m}^{2}}=-\frac{\left[W \sum_{i \in \mathbb{N}_{m}} \ln \left(1+r_{i}\right)\right]^{2}}{\left(\Gamma_{m}-\Gamma_{m}^{\min }\right)^{2}}<0 .
$$

The second derivative of $\ln U_{m}$ with respect to $\alpha_{m}$ is always negative. Therefore, $\ln U_{m}$ is concave in $\alpha_{m}$, and the problem (8) is a convex optimization problem. For a convex optimization problem, the optimal solution can be obtained by the Karush-Kuhn-Tucker (KKT) conditions. We give the Lagrange function.

$$
L\left(\alpha_{m}, \mu\right)=\sum_{m=1}^{M} \ln U_{m}-\mu\left(\sum_{m=1}^{M} \alpha_{m}-1\right)
$$

where $\mu$ is the Lagrange multiplier. We take the derivative of the Lagrange function with respect to $\alpha_{m}$ and $\mu$, respectively, and let them equal to zero.

$$
\left\{\begin{array}{l}
\mu=\frac{A_{m}}{\alpha_{m} A_{m}-\Gamma_{m}^{\min }} \\
\sum_{m=1}^{M} \alpha_{m}=1
\end{array}\right.
$$

from which, the optimal bandwidth allocation solution is obtained.

$$
\left\{\begin{aligned}
\mu^{*} & =\frac{M}{1-\sum_{m=1}^{M} \frac{\Gamma_{m}^{\min }}{A_{m}}} \\
\alpha_{m}^{*} & =\frac{\Gamma_{m}^{\min }}{A_{m}}+\frac{1-\sum_{m=1}^{M} \frac{\Gamma_{m}^{\min }}{A_{m}}}{M}
\end{aligned}\right.
$$

where $A_{m}$ is denoted as

$$
A_{m}=W \sum_{i \in \mathbb{N}_{m}} \ln \left(1+r_{i}\right)
$$

Thus, the corresponding net rate can be denoted as

$$
U_{m}=\frac{A_{m}\left(1-\sum_{m=1}^{M} \frac{\Gamma_{m}^{\min }}{A_{m}}\right)}{M} .
$$

The net rate (15) is related to the number of clusters and the minimum rates. Specifically, the net rate is gradually decreased with the minimum rates when the number of clusters is fixed.

\section{Numerical results}

We assume the femtocells are densely deployed in coverage of macrocell, and the radius of macrocell is $700 \mathrm{~m}$. We use $P_{a}$ to represent the percentage of active FBSs and investigate the performance with $P_{a}=40 \%$. Each active
FBS provides service to an FUE in one slot. The minimum separation distance between the FBS and the FUE is $3 \mathrm{~m}$. The penetration loss is set to $10 \mathrm{~dB}$. The parameters settings are given in Table 1.

Table 1 Simulation parameters

\begin{tabular}{lc}
\hline \multicolumn{1}{c}{ Parameters } & Settings \\
\hline Carrier frequency & $2 \mathrm{GHz}$ \\
Size of the apartment & $10 \mathrm{~m} \times 10 \mathrm{~m}$ \\
Cellular layout & $5 \times 5$ grid model \\
Bandwidth of one sub-channel & $10 \mathrm{MHz}$ \\
Transmission power of FBS & $13 \mathrm{dBm}$ \\
Path loss & $30 \log _{10} d+37$ \\
Penetration loss & $10 \mathrm{~dB}$ \\
Receiver noise figure & $9 \mathrm{~dB}$ \\
Minimum separation distance & $3 \mathrm{~m}$ \\
Percentage of active FBSs & $40 \%$ \\
\hline
\end{tabular}

Before clustering based on the weights, we need to know the value of $m$. The target SINR of FUEs is set to $20 \mathrm{~dB}$. In Fig. 3, we obtain the total net rates versus the number of clusters based on the Monte Carlo method. We find that the total net rates are maximal when $M$ is five.

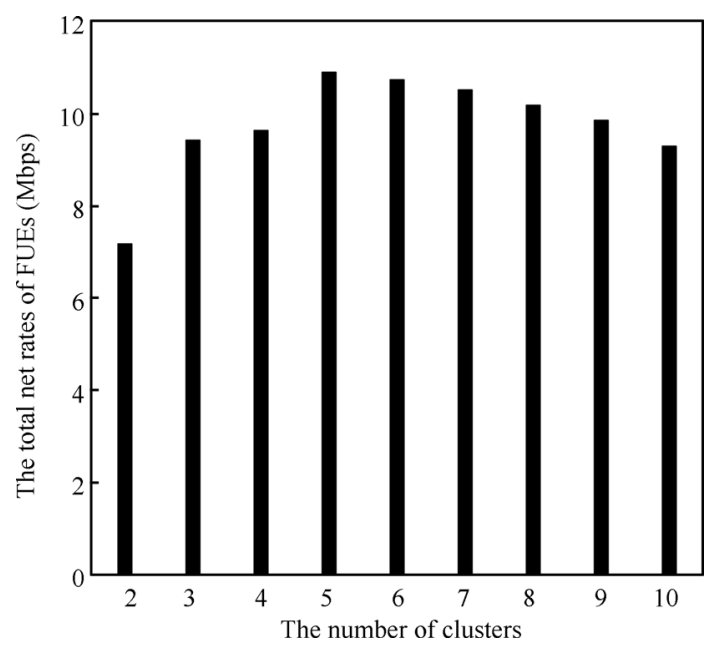

Fig. 3 The total net rates versus the number of clusters

The SINR of FUEs with and without clustering are shown in Fig. 4 when the number of clusters is set to 5 . We assume the target SINR is $20 \mathrm{~dB}$, which is the minimum SINR corresponding to the minimum rates of FUEs. We see that only three FUEs meet the target SINR without clustering and all of the FUEs meet the target SINR after clustering.

The optimal number of clusters versus the target SINR of FUEs is shown in Fig. 5. We see that the optimal number of clusters increases with the target SINR. Clustering is not needed when the target SINR is set to $5 \mathrm{~dB}$. The femtocells are divided into 10 clusters when the target SINR is higher than $40 \mathrm{~dB}$. The FUEs have achieved their maximal SINR when the target SINR is higher than $40 \mathrm{~dB}$. 


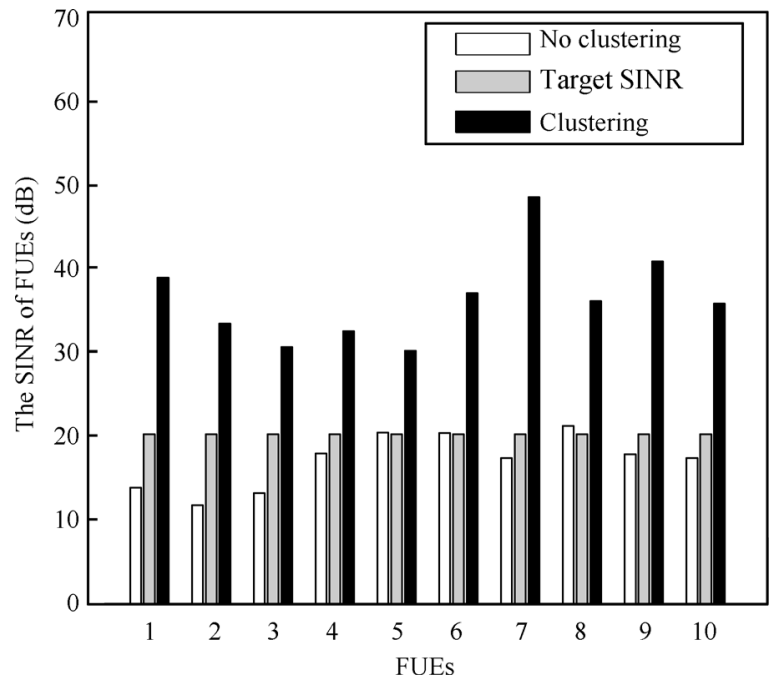

Fig. 4 The SINR of FUEs with and without clustering

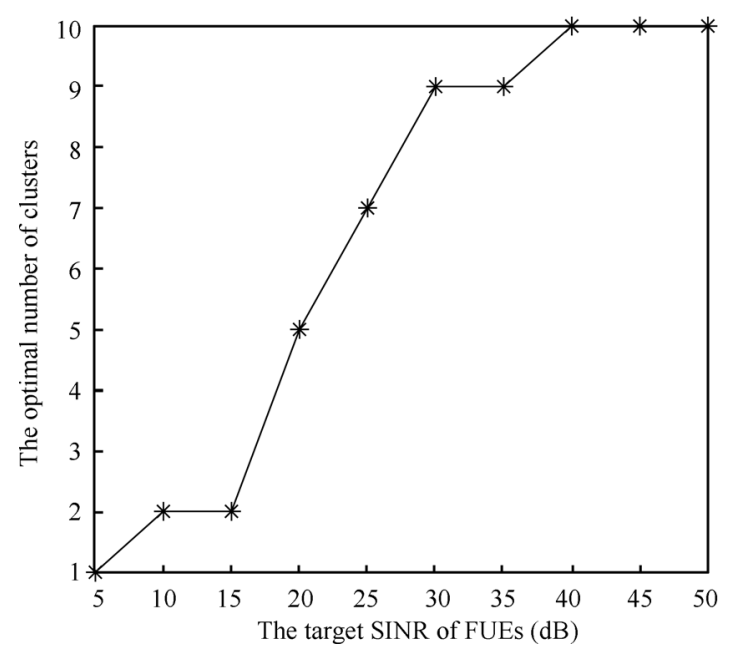

Fig. 5 The optimal number of clusters versus the target SINR of FUEs

The total net rates of FUEs versus the number of clusters are given in Fig. 6. The total net rates of FUEs increase at first and decrease with the number of clusters. We also compare the CBBAA with the average bandwidth allocation algorithm (ABAA). The ABAA outperforms the CBBAA when the number of clusters is less than 5. However, the CBBAA is much better when the number of clusters is more than 5. The FUEs cannot meet the total minimum rates when the number of clusters is 2 in both two bandwidths allocation schemes, and the total net rates of FUEs are maximal when the number of clusters is 5 .

The clustering results with different number of femtocells (NOF) are shown in Table 2. We give the number of clusters (NOC), the number of FUEs (NOE) in each cluster, and the fraction of bandwidth allocated (FBA) to each cluster. We see that the number of clusters is increased with the number of femtocells. Furthermore, an optimal fraction of bandwidth is allocated to each cluster. For example, the femtocells are divided into 11 clusters when the number of femtocells are 20. Although the allocated bandwidth of each cluster is small, the minimum rate constraint is satisfied and the fairness is achieved.

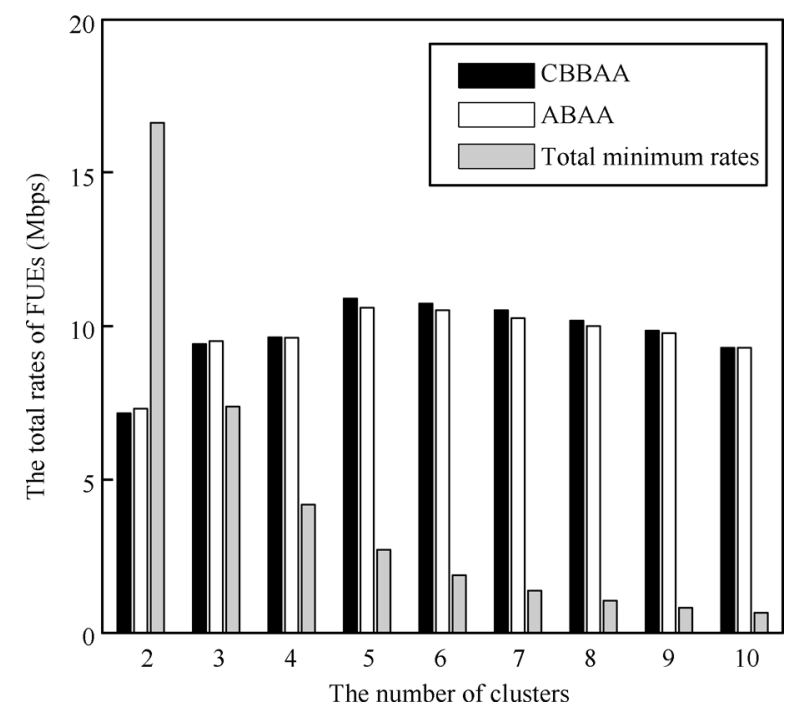

Fig. 6 The total net rates of FUEs versus the number of clusters

Table 2 The clustering results

\begin{tabular}{llll}
\hline NOF & NOC & NOE & FBA \\
\hline \multirow{3}{*}{5} & 3 & 1 & 0.30 \\
& & 2 & 0.36 \\
& 2 & 0.34 \\
\hline \multirow{2}{*}{10} & 5 & 2 & 0.20 \\
& & 3 & 0.22 \\
& & 2 & 0.19 \\
& & 1 & 0.17 \\
& & 2 & 0.21 \\
\hline \multirow{2}{*}{20} & 2 & 0.09 \\
& & 2 & 0.09 \\
& & 1 & 0.08 \\
& & 2 & 0.09 \\
& & 2 & 0.09 \\
& & 2 & 0.10 \\
& & 2 & 0.10 \\
& & 1 & 0.08 \\
& & 3 & 0.10 \\
& & 0.09 \\
& & 0.08 \\
\hline
\end{tabular}

\section{Conclusion}

In this paper, we study the inter-femtocell interference management problem when femtocells are densely deployed. We propose a cluster-based bandwidth allocation algorithm. Specifically, we construct the weighted interference graph and divide the femtocells into clusters. Then, the bandwidth is allocated to the clusters based on NBS. Simulation results show that the cluster-based bandwidth allocation can reduce the inter-femtocell interference and meet the minimum rate constraint of each cluster. 


\section{References}

[1] V. Chandrasekhar, J. G. Andrews, A. Gatherer. Femtocell networks: A survey. IEEE Communication Magazine, vol. 46, no. 9, pp. 59-67, 2008.

[2] V. Chandrasekhar, J. G. Andrews, T. Muharemovict, Z. K. Shen, A. Gatherer. Power control in two-tier femtocell networks. IEEE Transactions on Wireless Communications, vol. 8, no. 8, pp. 4316-4328, 2009.

[3] X. Kang, R. Zhang, M. Motani. Price-based resource allocation for spectrum-sharing femtocell networks: A stackelberg game approach. In Proceedings of IEEE Global Telecommunications Conference, IEEE, Houston, USA, pp. 1-5, 2011.

[4] M. S. Jin, S. A. Chae, D. I. Kim. Per cluster based opportunistic power control for heterogeneous networks. In Proceedings of the 73rd IEEE Vehicular Technology Conference, IEEE, Budapest, Hungary, pp. 1-5, 2011.

[5] K. Juyeop, C. H. Dong. A joint power and subchannel allocation scheme maximizing system capacity in indoor dense mobile communication systems. IEEE Transactions on Vehicular Technology, vol. 59, no. 9, pp. 4340-4353, 2010.

[6] S. Park, S. Bahk. Dynamic inter-cell interference avoidance in self-organizing femtocell networks. In Proceedings of IEEE International Conference on Communications, IEEE, Kyoto, Japan, pp. 1-5, 2011.

[7] L. Xue, X. P. Guan, Z. X. Liu, Q. C. Zheng. A power and coverage-aware clustering scheme for wireless sensor networks. International Journal of Automation and Computing, vol. 7, no. 4, pp. 500-508, 2010.

[8] W. Li, T. Su, W. Zheng, X. M. Wen. Clustering based resource allocation for inter-femtocell interference management. Journal of Computation Information Systems, vol. 8, no. 4, pp. 1457-1466, 2012.

[9] A. Hatoum, N. Aitsaadi, R. Langar, R. Boutaba. FCRA: Femtocell cluster-based resource allocation scheme for OFDMA networks. In Proceedings of IEEE International Conference on Communications, IEEE, Kyoto, Japan, pp. 1-6, 2011.

[10] K. Ma, X. P. Guan, J. Wang, X. M. Wei. Multi-user symmetric cooperation based on Nash bargaining solution in cooperative relay networks. International Journal of $\mathrm{Au}$ tomation and Computing, vol. 8, no. 4, pp. 452-458, 2011.

[11] D. Fudenberg, J. Tirole. Game Theory, Cambridge, MA, USA: MIT, 1991.

[12] K. Ma, X. P. Guan, B. Zhao, J. Wang. A cooperation strategy based on bargaining solution in wireless sensor networks. Journal of Control Theory and Applications, vol.9, no. 1, pp. 121-126, 2011.

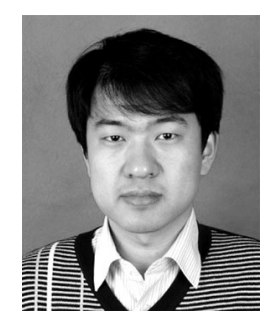

Kai Ma received his B. Sc. degree in automation and Ph. D. degree in control science and engineering from Yanshan University, China in 2005 and 2011, respectively. In 2011, he joined Yanshan University. From 2013 to 2014, he has been a postdoctoral research fellow in Nanyang Technological University, Singapore. He is currently an associate professor with the Department of Automation, School of Electrical Engineering, Yanshan University, China. He has published more than 30 refereed journal and conference papers.

His research interests include resource allocation in communication networks and demand response in smart grid.
E-mail: kma@ysu.edu.cn (Corresponding author) ORCID iD: 0000-0003-3517-5620

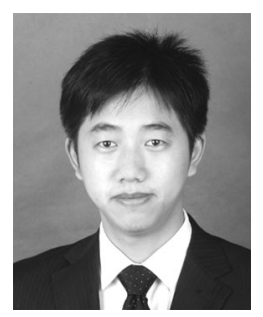

Liang $\mathbf{L i}$ received his B. Sc. degree in automation from $\mathrm{Xi}^{\prime}$ an Technological University, China in 2011. He is currently pursuing his master degree in the Department of Automation at Yanshan University, China.

His research interest includes femtocell networks.

E-mail: 906496696@qq.com

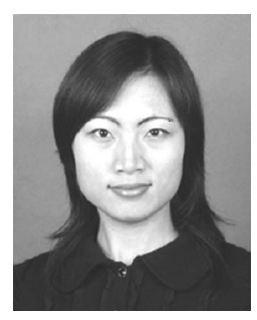

Jie Yang received her B.Sc. degree in electrical engineering and M. Sc. degree in control theory and control engineering from Yanshan University, China in 2006 and 2009, respectively.

Her research interests include time-delay systems and demand response in smart grid.

E-mail: jyang.tju@gmail.com

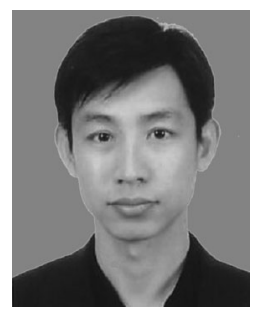

Zhi-Xin Liu received his B.Sc., M. Sc. and Ph. D. degrees in control theory and engineering from Yanshan University, China in 2000,2003 , and 2006, respectively. He is currently a professor with the Department of Automation, School of Electrical Engineering, Yanshan University, China. He visited the University of Alberta, Edmonton, Alberta, Canada in 2009.

His research interests include performance optimization and energy-efficient protocol design in wireless sensor networks and wireless resource allocation in cognitive radio networks.

E-mail: lzxauto@ysu.edu.cn

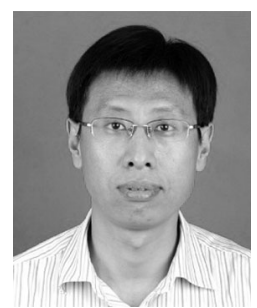

Xin-Bin Li received his M. Sc. degree in control theory and control engineering from Yanshan University, China in 1999, and his Ph. D. degree in general and fundamental mechanics from Peking University, China in 2004. He is currently a professor in the School of Electrical Engineering, Yanshan University.

His research interests include nonlinear system and networked control system.

E-mail: lixb@ysu.edu.cn

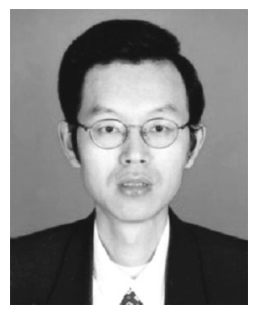

Xin-Ping Guan received his M. Sc. degree in applied mathematics from Harbin Institute of Technology, China in 1991, and his $\mathrm{Ph} . \mathrm{D}$. degree in electrical engineering from Harbin Institute of Technology, China in 1999. He is a professor with Department of Automation, Shanghai Jiao Tong University, China.

His research interests include functional differential and difference equations, robust control and intelligent control of time-delay systems, chaos control and synchronization, and congestion control of networks.

E-mail:xpguan@sjtu.edu.cn 